\title{
Evaluation of the Pharmacologic Treatment of COVID-19 Pandemic in Iraq
}

\author{
Omeed Darweesh ${ }^{1} \cdot$ Ghayth M. Abdulrazzaq ${ }^{2} \cdot$ Radhwan N. Al-Zidan $^{2}$ (D) $\cdot$ Pshtiwan Bebane $^{3} \cdot$ Marwan Merkhan $^{4}$. \\ Ruya Aldabbagh ${ }^{5} \cdot$ Nohad AlOmari $^{6}$ (D)
}

Accepted: 29 June 2021 / Published online: 5 August 2021

(C) The Author(s), under exclusive licence to Springer Nature Switzerland AG 2021

\begin{abstract}
The impact of severe acute respiratory syndrome coronavirus 2 (SARS-CoV-2, or COVID-19) has been detrimental to human health, economy, and wellbeing. Little information is known on the epidemiology and outcome of the disease in a localized community within Iraq. We carried out an audit of confirmed cases of COVID-19 in the Kirkuk General Hospital. Data from the 20th of June to the 31st of July, 2020, were collected and analyzed. Suspected COVID-19 cases were confirmed by real-time polymerase chain reaction (RT-PCR). Data on clinical symptoms, age, and treatment protocols were analyzed concerning the outcome. Our study included a total of 200 individual confirmed COVID-19 patients. The majority of cases 55\% $(n=110)$ displayed severe symptoms, while $32.5 \%$ ( 65 cases) and $12.5 \%$ ( 25 cases) of patients displayed moderate to mild symptoms, respectively. The rate of death in the referred patients was 5\%. Most patients admitted to the hospital for treatment recovered and were discharged from the hospital within 5 to 30 days post-diagnosis. Statistical analysis revealed that patients treated with oseltamivir, hydroxychloroquine, and azithromycin in combination with vitamins $\mathrm{C}$ and $\mathrm{D}$ have shorter hospital stay compared to patients receiving the same therapeutic protocol in combination with steroids. Moreover, a higher mortality rate (4.5\%) was observed in patients treated with oseltamivir, hydroxychloroquine, ceftriaxone, and steroids. This study highlights a significant relationship between age, secondary ailments, and the choice of medications as simple predictors of the outcome of COVID-19.
\end{abstract}

Keywords COVID-19 $\cdot$ Clinical features $\cdot$ Treatment strategies $\cdot$ Hospitalization stay $\cdot$ Mortality

\section{Introduction}

First reported on the 26th of December 2019 as irregular pneumonia in 4 Chinese patients, SARS-CoV-2 was soon declared as "Public Health Emergency of International Concern" by the World Health Organization (WHO) on the 30th of January, and subsequently as a "global pandemic" on the 11 th of

This article is part of the Topical Collection on Clinical Pharmacology

Nohad AlOmari

nohad.alomari@knu.edu.iq

College of Pharmacy, Al-kitab University, Kirkuk, Iraq

2 College of Pharmacy, University of Mosul, Mosul, Iraq

3 College of Education, Charmo University, Chamchamal, Iraq

4 College of Pharmacy, Ninevah University, Mosul, Iraq

5 Kirkuk Health Directorate, Ministry of Health, Kirkuk, Iraq

6 College of Pharmacy, Knowledge university, Erbil, Iraq
March 2020 [1]. Nations around the world have been stricken by the SARS-CoV-2, which is the strain of coronavirus that causes the COVID-19 outbreak, and Iraq has not been spared. The rapidly increasing cases of COVID-19 have been alarming in neighboring countries, particularly in Iran. Therefore, the Iraqi government imposed several health measures on domestic and international traveling. For instance, travelers arriving from the COVID-19 outbreak area are quarantined for at least 14 days and screened for the SARS-CoV-2 virus by the PCR test. Additionally, before the end of February 2020, the schools and universities have been closed. Furthermore, on the 13th of March 2020, the curfew was imposed for more than 1 month, and intermittently onward [2].

Patients with COVID-19 infection usually present with tiredness, headache, fever, dry cough, breathlessness, and/or pneumonia, while loss of taste or smell, diarrhea, productive cough, and sore throat are less frequent $[3,4]$. The infection is caused by a novel virus with a clinical pattern that is not fully understood. Therefore, since the start of the COVID-19 
pandemic, several experimental drugs were suggested and used [5]. Lastly, as of February 08, 2021, the total number of the confirmed COVID-19 cases exceeded 106 million, whereas the death toll more than 2.3 million, worldwide [6]. Hence, the Iraqi Ministry of Health (MOH), like all the counterpart agencies around the globe, adopted and frequently updated treatment protocols for the management of the clinical condition of the COVID-19 patients in an endeavor to reduce the morbidity and mortality rate. For instance, Allawi's study, which was conducted in Baghdad's Medical City Teaching Hospital (MCTH) in April 2020, reported the first protocol adopted by the Iraqi $\mathrm{MOH}$ [7]. The recommendations for the care of COVID-19 patients vary widely, especially during the early months of the pandemic, between the different countries around the world. While there is no approved medication for COVID-19, the general guidance is to provide supportive management as per the needs of each patient, for instance, the saturated $\mathrm{O}_{2}$ (particularly in patients with $\mathrm{SpO} 2$ lower than $90 \%$ ), the immune-boosters (such as vitamins $\mathrm{C}$ and $\mathrm{D}$ and zinc), and the antipyretics (such as acetaminophen) for fever [8]. To counteract the vicious pandemic, numerous clinicians and scientists around the globe suggested the use of different medications that are believed to have a potential antiviral action against the COVID-19 virus, such as hydroxychloroquine, favipiravir, remdesivir, or azithromycin $[8,9]$. On the other hand, mounting evidence was suggesting that patients with a rapidly deteriorating condition might be suffering from a severe autoimmune reaction. Therefore, immunomodulating drugs, such as dexamethasone, anakinra, and tocilizumab, were suggested to be used in patients with severe COVID-19 infections [8].

Currently, there is a large volume of research being conducted to understand the pathophysiology and clinical characteristics of COVID-19 [10,11], patients' demographics (e.g., age and sex) $[12,13]$, and the impact of different treatment protocols on the morbidity and mortality $[9,14]$. However, to the best of our knowledge, no studies from Iraq have been published to cover these pivotal aspects. Therefore, we are presenting the first study from Kirkuk province to scrutinize the nature of the COVID-19 cases and to evaluate the impact of the adopted treatment protocols on the treated sample of the COVID-19 patients.

\section{Methods}

In this descriptive, single-center study, we recruited patients with confirmed COVID-19 infection. All of the patients were tested positive by real-time reverse transcription-polymerase chain reaction (RT-PCR), testing of a nasopharyngeal swab sample. The samples were immediately maintained in a viral transport medium and were tested to confirm COVID-19 using Bosphore Novel Coronavirus (2019-nCoV) Detection Kit. The patients were admitted to Kirkuk General Hospital, which follows the protocol of the Iraqi Ministry of Health for treating the COVID-19 patients, from the 20th of June to the 31 st of July, 2020. This study was approved by the ethics committee of the Kirkuk Health Directorate. All of the COVID-19 patients enrolled in this study were diagnosed according to the WHO guidelines.

Clinical data of 200 COVID-19 patients were recorded during hospital admission and treatment tours from the 20th of June 2020 to the final date of follow-up. Physicians and other medical workers from the emergency department, the internal medicine department, critical care unit, and pharmacy unit were involved in the diagnosis and treatment team. Statistical analysis was conducted using R studio v 1.3.1093. One-way analysis of variance was used for multiple group comparisons, and Tukey's post hoc test was used for intergroup comparisons. $p$ adj $<0.05$ was considered to indicate a statistically significant difference.

\section{Results}

Based on WHO criteria, patients were classified into three main groups on the day of admission (Fig. 1). Mild disease meets the case definition for COVID-19 without evidence of hypoxemia or pneumonia. In moderate cases, patients display clinical signs of pneumonia, like fever, cough, dyspnea, and fast breathing with $\mathrm{SpO} 2$ equal to or more than $90 \%$ on room air. The severe disease is defined as the patients displaying clinical features of pneumonia plus either of the following: respiratory rate that is more than 30 breaths/min or SpO2 less than $90 \%$ on room air. Out of the 200 confirmed COVID-19 patients, $110(55 \%)$ cases were admitted as severe, $65(32.5 \%)$ cases were admitted as moderate, and only $25(12.5 \%)$ of the cases were mild (Fig. 1). The mean age of our study population was 43.3 years $( \pm 16.8)$, including $62.5 \%$ men and $37.5 \%$ women. In the present study, 22 years of age was observed as the youngest and 90 years of age as the oldest patient within our cohort. The oldest patients included in all protocols

Table 1 Age of patients sub-classified according to the used protocols

\begin{tabular}{lrrrrr}
\hline Protocol & \multicolumn{2}{l}{ Age (years) } & & \multicolumn{2}{l}{ Sex } \\
\cline { 2 - 3 } \cline { 6 - 6 } & Mean \pm SD & Range & & Male & Female \\
\hline Protocol 1 & $39.5 \pm 16.6$ & $22-78$ & & 19 & 6 \\
Protocol 2 & $47.2 \pm 11.9$ & $35-70$ & & 20 & 6 \\
Protocol 3 & $39.4 \pm 14.9$ & $27-77$ & & 30 & 29 \\
Protocol 4 & $38 \pm 14.9$ & $26-70$ & & 28 & 24 \\
Protocol 5 & $58.4 \pm 15.9$ & $25-90$ & & 28 & 10 \\
Overall & $43.3 \pm 16.8$ & $22-90$ & & 125 & 75 \\
\hline
\end{tabular}


were in their 70s; except those used in protocol 5, their age ranged in their early 90 s years (Table 1 ).

The treatment protocols of COVID-19 patients are presented in Tables 2 and 3. The treatment protocols were adopted by senior healthcare professionals in Kirkuk General Hospital in agreement with the protocols approved by the Iraqi Ministry of Health. These protocols have been selected based on patients' condition (age, sign, and symptoms, and $\mathrm{SpO} 2$ upon admission). The cornerstone of the protocol was based on the selection of oseltamivir, hydroxychloroquine, azithromycin, and ceftriaxone. In addition to the aforementioned therapies, supportive therapy was included in the study. This consisted of the administration of vitamins $\mathrm{C}$ and $\mathrm{D}$ and zinc and the antipyretic medication, paracetamol. Moreover, dexamethasone was added to treatment protocols 4 and 5 according to the severity of the patient's symptoms. The mild disease which was reported in $12.5 \%$ of the cases was treated by "protocol 1" which included paracetamol plus vitamins C, D and zinc. Patients with the moderate and severe diseases phenotype proportionally received the antiviral therapy Oseltamivir in addition to receiving anti-bacterial therapy including azithromycin or ceftriaxone. Regarding corticosteroids, dexamethasone was only used in severe cases, which were treated with protocols 4 and 5 (Tables 2 and 3).

Among the patients, 110 cases met the criteria for severe COVID-19-associated pneumonia, and the majority of these cases were treated using protocols 4 and 5 (Table 4). However, most of the moderate cases were treated with protocols 2 and 3. In terms of patient number, there were circa half the cases of severe than moderate (Table 4). Patients treated with protocols 1 and 2 had comparable hospitalization stay $11.1( \pm 1.61)$ and $12.5( \pm 3.14)$ respectively (Fig. 2). Patients treated with protocol 3 had more length of hospital stay of $14.5( \pm 2.22)$. However, a higher hospital stay time was reported in patients treated with protocols 4 and 5, 17.5 $( \pm 4.04)$ and $19.3( \pm 3.86)$, respectively (Fig. 2). However, there was no significant difference in the length of hospital stay between protocols 1, 2, and 3. The length of hospital stay was significantly higher in patients treated with protocols 4 and 5 compared to protocols $1-3(p<0.01)$. To determine whether there was any correlation between treatment protocols and hospitalization stay in terms of patients' gender. Hospitalization stay of female patients was similar to all patients in different treatment protocols (Fig. 3). Patients treated with protocols 4 and 5 had a significantly higher length of hospital stay compared to protocols 1,2 , and $3(p<0.01)$. Identical results were obtained in male patients (Fig. 4). Furthermore, there was no significant difference in the length of hospital stay in male patients compared to females, 15.2 $( \pm 4.06)$ and $15.9( \pm 4.44)$ respectively (Fig. 5). A total of 190 patients had been successfully discharged, and 10 patients had unfortunately died. Most death cases were recorded in patients treated with protocol 5 , nine death cases. One death was reported in patients treated with protocol 3. The most prevalence of comorbidity among death cases was hypertension (4 cases), which followed by diabetes mellitus ( 2 cases), and cardiovascular disease ( 2 cases). The mean age of death cases was 75.3 years, ranging from 50 to 90 years. The overall mortality rate was $5 \%$ in the entire study. The mortality rate was higher in male patients compared to females, $4.5 \%$ and $0.5 \%$ respectively.

Oxygen saturation $(\mathrm{SpO} 2)$ values in different age groups showed that elderly patients are more vulnerable to low $\mathrm{SpO} 2$ and younger patients had the capacity to tolerate the disease more effectively, displaying higher values of oxygen saturation (Fig. 6). The mean $\mathrm{SpO} 2$ in patients of 60 years and above was 88.6. However, $\mathrm{SpO} 2$ was higher than 90 in patients younger than 60 years. Patients with mild symptoms treated with protocol 1 showed higher $\mathrm{SpO} 2$ values, $94.72( \pm 1.54)$, ranging from 93.00 to 98.00 (Fig. 6). $\mathrm{SpO} 2$ values in patients treated with protocol 1 were significantly higher compared to protocols $2-5(p<0.01)$. All the COVID-19 patients treated
Fig. 1 Admitted patients were sub-classified into mild, moderate, and severe based on WHO criteria on patient symptoms upon hospital admission

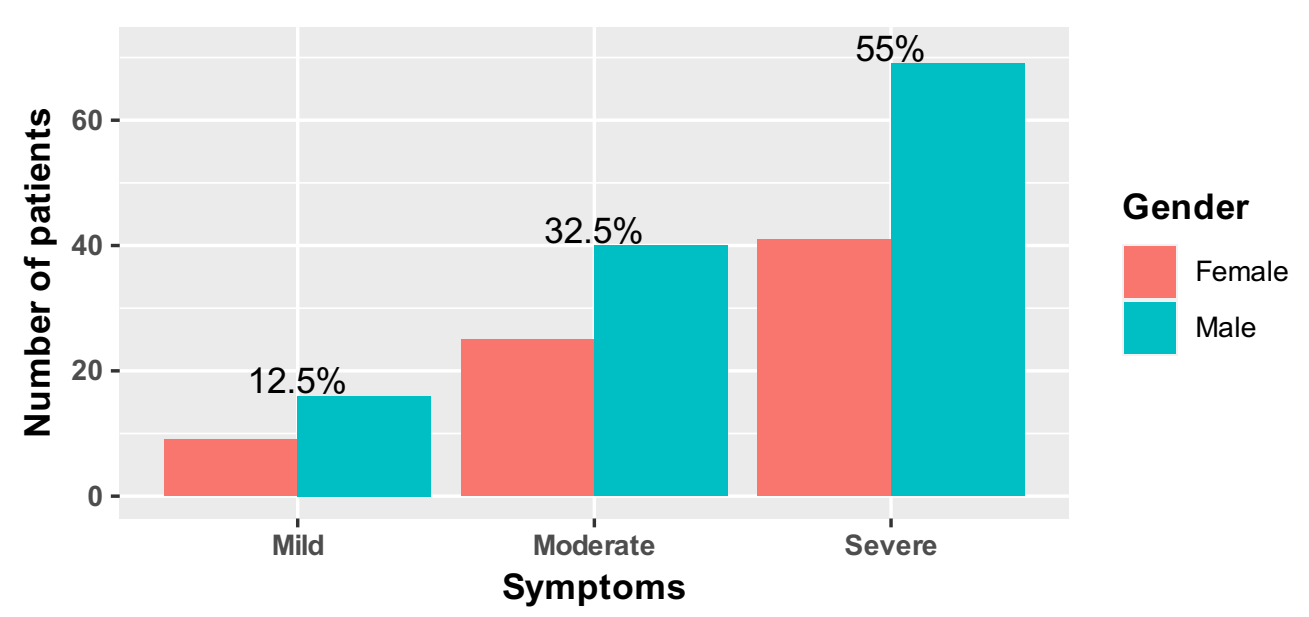


Table 2 Protocols adopted by healthcare professionals in Kirkuk General Hospital

Protocol No. Oseltamivir Azithromycin Hydroxychloroquine Ceftriaxone Dexamethasone Paracetamol Vitamin C Vitamin D Zinc

\begin{tabular}{|c|c|c|c|c|c|c|c|c|c|}
\hline Protocol 1 & & & & & & $*$ & $*$ & $*$ & $*$ \\
\hline Protocol 2 & $*$ & $*$ & & & & $*$ & $*$ & $*$ & $*$ \\
\hline Protocol 3 & $*$ & $*$ & $*$ & & & $*$ & $*$ & $*$ & * \\
\hline Protoco 14 & $*$ & & & $*$ & $*$ & $*$ & $*$ & $*$ & $*$ \\
\hline Protocol 5 & $*$ & & $*$ & $*$ & $*$ & $*$ & $*$ & $*$ & $*$ \\
\hline
\end{tabular}

Table 3 COVID-19 treatment protocol

\begin{tabular}{ll}
\hline Clinical presentation & Treatment protocol \\
\hline Mild & Protocol 1 \\
Moderate & Oseltamivir $75 \mathrm{mg}$ orally twice a day for 5 days \\
& Azithromycin $500 \mathrm{mg}$ daily for 6 days \\
& Hydroxychloroquine $400 \mathrm{mg}$ twice daily first day then $200 \mathrm{mg}$ twice daily for 7 days \\
& Ceftriaxone $1 \mathrm{~g}$ daily for 7 days \\
& Oseltamivir $75 \mathrm{mg}$ orally twice a day for 5 days \\
Severe & Azithromycin $500 \mathrm{mg}$ daily for 6 days \\
& Hydroxychloroquine $400 \mathrm{mg}$ twice daily first day then $200 \mathrm{mg}$ twice daily for 14 days \\
& Ceftriaxone $1 \mathrm{~g}$ twice daily for 14 days \\
& Dexamethasone $6 \mathrm{mg}$ per day up to 10 days
\end{tabular}

with protocols 2, 3, 4, and 5 with $\mathrm{SpO} 2$ values less than ninety received supplemental oxygen. Patients treated with protocols 2, 3, and 4 displayed comparable $\mathrm{SpO} 2$ values, $90.2( \pm 3.67)$, ranging from 83.00 to $97.00,89.7( \pm 3.44)$, ranging from 83.00 to $95.00,88.3( \pm 4.00)$, and ranging from 81.00 to 95.00 , respectively. However, lowest $\mathrm{O} 2$ saturation was recorded in protocol $5(86.6( \pm 5.06)$, ranging from 75.00 to 94.00). Therefore, patients treated with protocol 5 had a significantly lower SpO2 compared to protocols 2 and 3 $(p<0.01)$. SpO2 values in different used protocols showed that patients on protocol 5 had the lowest $\mathrm{O} 2$ saturation and patients on protocol 1displayed a higher level of $\mathrm{SpO} 2$ (Fig. 7).

Table 4 Classification of patients according to their symptoms upon admission and the used protocol

\begin{tabular}{llll}
\hline Protocol number & \multicolumn{2}{l}{ Severity of symptoms on admission } \\
\cline { 2 - 4 } & Mild & Moderate & Severe \\
\hline Protocol 1 & 25 & 0 & 0 \\
Protocol 2 & 0 & 19 & 15 \\
Protocol 3 & 0 & 25 & 17 \\
Protocol 4 & 0 & 12 & 50 \\
Protocol 5 & 0 & 9 & 28 \\
Total & 25 & 65 & 110 \\
\hline
\end{tabular}

\section{Discussion}

The SARS-CoV-2 pandemic infection has become a global health issue since it has been described for the first time in December 2019 in Wuhan, China. This study was conducted on COVID-19 infected patients in Kirkuk Province as a representative sample for patients infected with SARS-CoV-2 in Iraq. In this study, we followed up 200 COVID-19-infected patients of age range between 22 and 90 years with disease severity varies from mild through moderate to severe illness. Mild cases were admitted and treated at the hospital until full recovery according to a strategy of hospital isolation of all suspected and confirmed individuals that was adopted by the Ministry of Health in Iraq to reduce the local transmission of SARS-CoV-2. More than half of the patients included in our study were severely ill (Fig. 1) and the majority of the reported cases of death were in old age male patients who showed a marked reduction in oxygen saturation and were severely ill on hospital admission (see Figs. 6 and 7). Male gender appears to be associated with severe illness and a higher rate of death in the present study. This finding is consistent with previous studies from China suggesting a higher fatality rate among men in comparison with women [15-18]. Yet, the role of underlying conditions that caused the death could not be properly assessed in our study because of difficulty in accessing the required information. Despite that, the higher prevalence of critical illness and deaths in the male rather than female 
Fig. 2 Length of hospital stay (days) for all patients according to treatment protocols

Fig. 3 Length of hospital stay (days) for female patients according to treatment protocols

Fig. 4 Length of hospital stay (days) for male patients according to treatment protocols
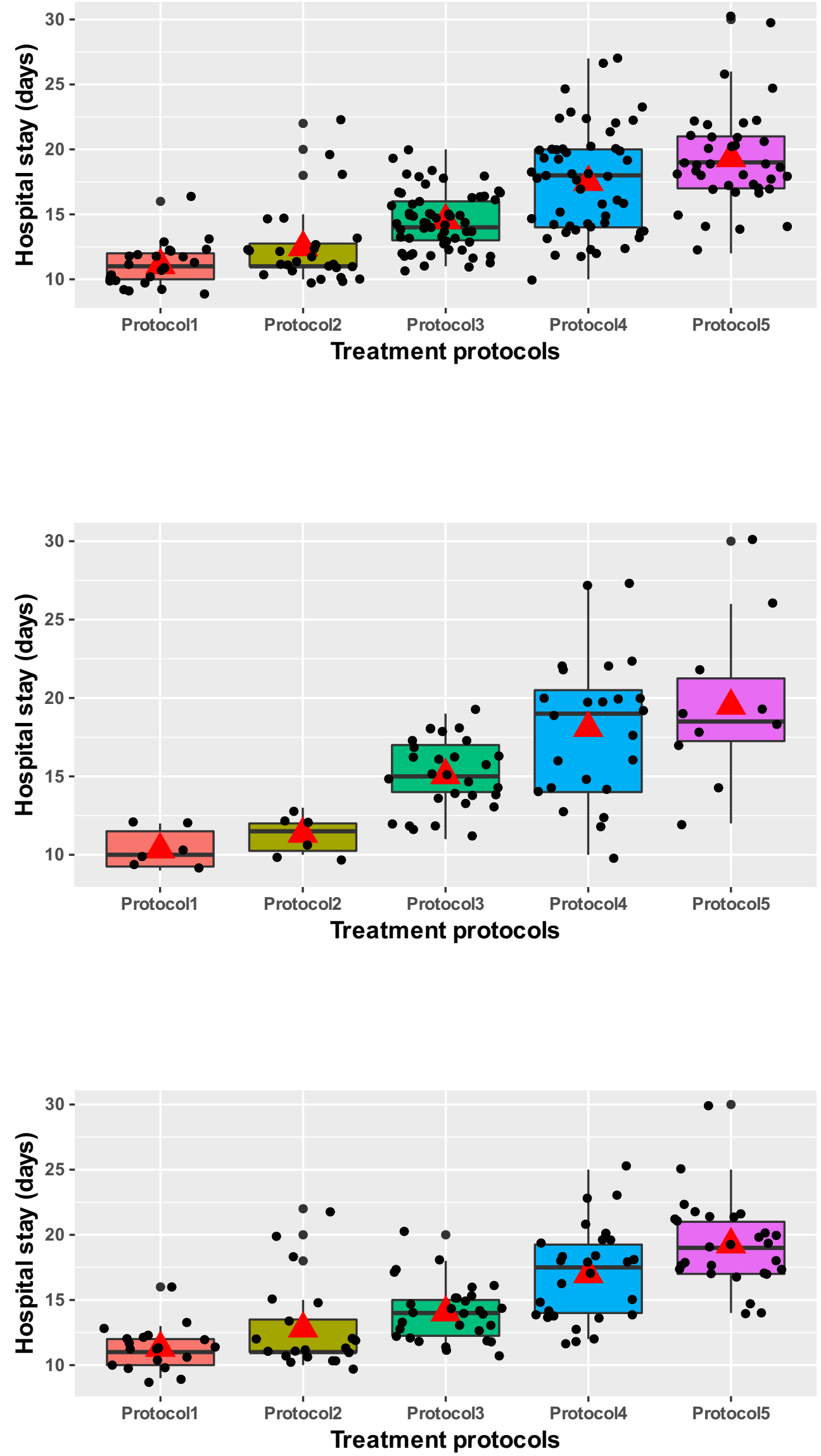
Fig. 5 Length of hospital stay (days) for female and male patients

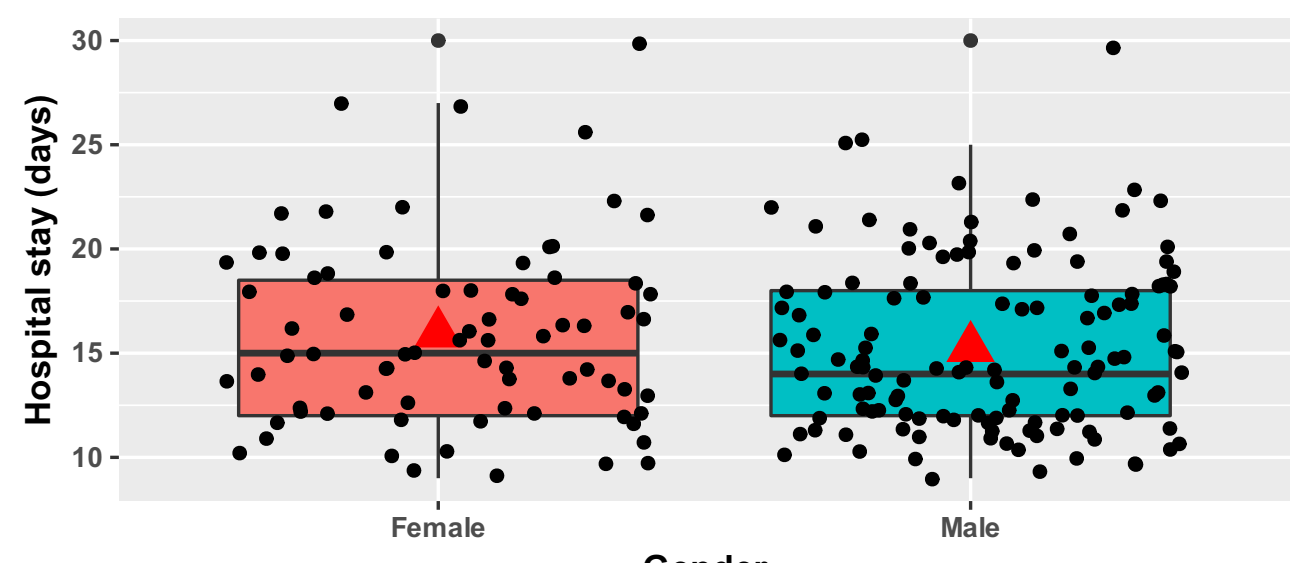

Gender gender had been attributed to the higher level of expression of the viral entry protein, angiotensin-converting enzyme-2 (ACE2) by respiratory epithelial cells in men $[19,20]$. However, the direct cause of death is yet to be determined. Generally, men are more likely to have perilous and unhealthy behaviors than women, such as their higher smoking rate, which makes them more vulnerable to the risk of fatality. Co-existing chronic diseases may also contribute to the increased morbidity and mortality in male and female patients with coronavirus infection [21].

The efficiency of five different therapeutic protocols adopted by healthcare system in Iraq (Table 1) in reference to the WHO guidelines for the management of COVID-19 infection (Table 2) was assessed and compared in the current study. We reported that the inclusion of steroids in the therapeutic regimen is associated with prolonged hospital admission in a male (Fig. 4) and female (Fig. 3) patients. The pattern of response appears to be similar in male and female patients (Fig. 5). This effect was particularly prominent and shown to be associated with increased frequency of deaths when steroids co-administered with hydroxychloroquine and azithromycin as in protocol 5 .
Several reports published recently might support the potential therapeutic benefits of using corticosteroids in the treatment regimen of SARS-CoV-2 infection. Some researchers correlate a low incidence of COVID-19 with long-term use of steroids as in patients with rheumatic diseases [22] kidney transplant recipients [23]. Other researchers have even proposed corticosteroids as a potential therapeutic medicine in SARS-CoV-2 infection [24, 25]. However, the data presented in these studies are incomplete or inconsistent on study design and outcomes. With this in mind, it was difficult to get a conclusive idea about the efficacy and safety of steroids for COVID-19 infection.

Corticosteroids are not recommended in most COVID-19 infection management guidelines. In the absence of conclusive scientific evidence, WHO and the US Centers for Disease Control and Prevention (CDC) have recommended that corticosteroids should not be used routinely in patients with COVID-19 for treatment of viral pneumonia or acute respiratory distress syndrome (ARDS) unless indicated for other conditions, such as asthma or chronic obstructive pulmonary disease exacerbation, or septic shock [26-28]. Careful use of low-to-moderate doses of corticosteroids as a short course is advised.
Fig. 6 Correlation between age and oxygen saturation in hospitalized COVID-19 patients

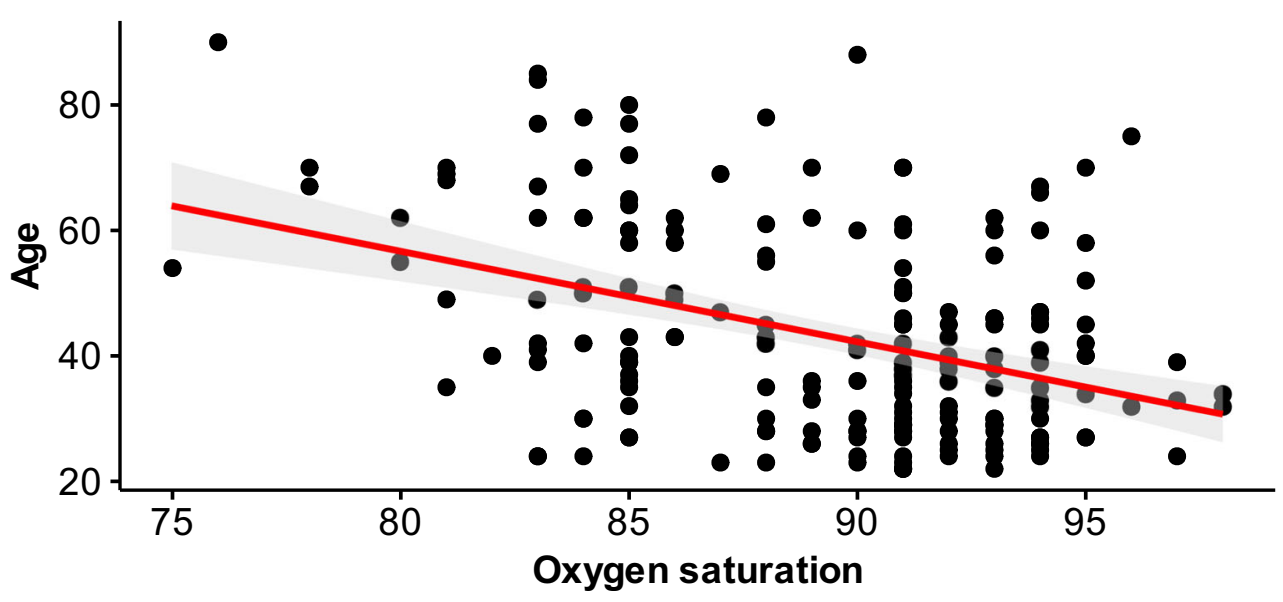


Fig. 7 Oxygen saturation in hospitalized COVID-19 patients according to treatment protocols

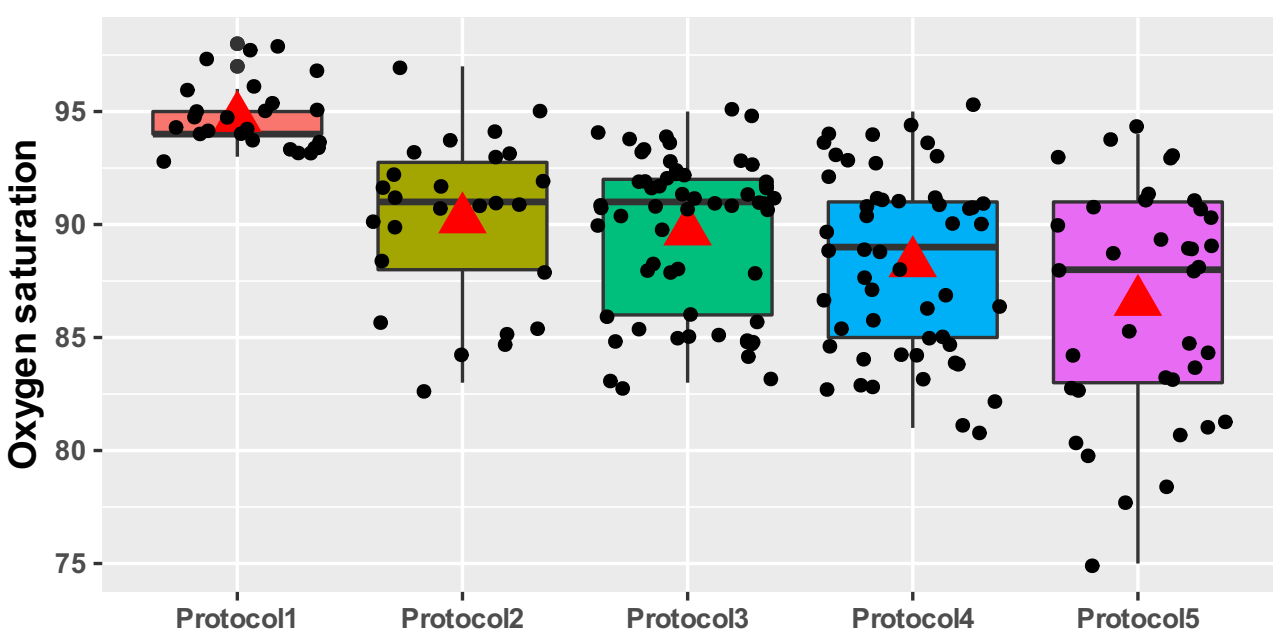

Treatment protocols
Hyperglycemia, hypernatremia, and hypokalemia are the most common adverse effects associated with the use of corticosteroids and should be monitored routinely [28].

Our study has several limitations, this may include that the observational design of the study that halt causal inference. Yet, the situation of such public health emergency makes it very difficult to conduct a comprehensive study. Also, the duration of follow-up was relatively short. Moreover, some data were obtained on the basis of self-report and we could not verify their validity. However, access to such information is strictly regulated by higher authorities. We are looking forward to getting official permission to obtain updated and more inclusive data for further research.

In conclusion, robust and timely epidemiological measures are imperative to control the rapid spread of the COVID-19 pandemic. This study highlighted the gender difference in susceptibility to infection by SARS-CoV-2 and efficiency of therapeutic protocols for management of COVID-19 and its related mortality, suggesting that male gender and probably chronic use of steroids may cause more critical illness and a higher risk of fatality. Comparison between the efficiency of several therapeutic protocols in the present study could be helpful for physicians and healthcare providers in the selection of appropriate therapeutic regimens.

Acknowledgments The authors thank the administration board of Kirkuk General Hospital for cooperation and help in collecting the research data.

Author Contribution Study design: Omeed Darweesh and Nohad AlOmari

Data collection: Omeed Darweesh and Ruya Aldabbagh

Data analysis and visualization: Pshtiwan Bebane, Omeed Darweesh, and Marwan Merkhan

Writing original draft: Omeed Darweesh, Radhwan Al-Zidan, Ghayth Abdulrazzaq, Marwan Merkhan, and Nohad AlOmari

Reviewing and editing: Omeed Darweesh, Radhwan Al-Zidan, Ghayth Abdulrazzaq, and Nohad AlOmari

\section{Declarations}

Conflict of Interest The authors declare that they have no competing interests.

Human and Animal Rights and Informed Consent This article does not contain any studies with human or animal subjects performed by any of the authors.

Ethics Approval Kirkuk Health Directorate Human Ethics Committee approved the protocol (ethical approval number 15549).

Patient Consent for Publication All participants gave informed consent.

\section{References}

1. Cucinotta D, Vanelli M. WHO declares COVID-19 a pandemic. Acta Bio Medica: Atenei Parmensis. 2020;91(1):157-60.

2. Organization, W.H. Laboratory assessment tool for laboratories implementing COVID-19 virus testing, 08 April 2020: World Health Organization; 2020.

3. Adhikari SP, et al. Epidemiology, causes, clinical manifestation and diagnosis, prevention and control of coronavirus disease (COVID19) during the early outbreak period: a scoping review. Infect Dis Poverty. 2020;9(1):1-12.

4. Huang C, et al. Clinical features of patients infected with 2019 novel coronavirus in Wuhan, China. Lancet. 2020;395(10223): 497-506.

5. Iacob S, Iacob DG. SARS-coV-2 treatment approaches: numerous options, no certainty for a versatile virus. Front Pharmacol. 2020;11:1224

6. Dong E, Du H, Gardner L. An interactive web-based dashboard to track COVID-19 in real time. Lancet Infect Dis. 2020;20(5):533-4.

7. Allawi JS, et al. The first 40-days experience and clinical outcomes in the management of coronavirus covid-19 crisis. Single center preliminary study. J Fac Med Baghdad. 2019;61(3, 4).

8. Peng Y, et al. A comprehensive summary of the knowledge on COVID-19 treatment. Aging Dis. 2021;12(1):155.

9. Gudadappanavar AM, Benni J. An evidence-based systematic review on emerging therapeutic and preventive strategies to treat novel coronavirus (SARS-CoV-2) during an outbreak scenario. J Basic Clin Physiol Pharmacol. 2020;31(6). 
10. Tsatsakis A, et al. SARS-CoV-2 pathophysiology and its clinical implications: an integrative overview of the pharmacotherapeutic management of COVID-19. Food Chem Toxicol. 2020;146: 111769.

11. Khan M, et al. Epidemiological and clinical characteristics of coronavirus disease (COVID-19) cases at a screening clinic during the early outbreak period: a single-centre study. J Med Microbiol. 2020;69(8):1114.

12. Peckham $\mathrm{H}$, et al. Male sex identified by global COVID-19 metaanalysis as a risk factor for death and ITU admission. Nat Commun. 2020;11(1):1-10.

13. Jin J-M, et al. Gender differences in patients with COVID-19: focus on severity and mortality. Front Public Health. 2020;8:152.

14. Al-Zidan RN. Potential drug-drug and drug-disease interactions of selected experimental therapies used in treating COVID-19 patients. J Drug Deliv Ther. 2020;10(6):219-30.

15. Pan A, et al. Association of public health interventions with the epidemiology of the COVID-19 outbreak in Wuhan, China. Jama. 2020;323(19):1915-23.

16. Wang F, et al. Epidemiological characteristics of patients with severe COVID-19 infection in Wuhan, China: evidence from a retrospective observational study. Int J Epidemiol. 2020;49(6):1940-50.

17. Yang X, et al. Clinical course and outcomes of critically ill patients with SARS-CoV-2 pneumonia in Wuhan, China: a single-centered, retrospective, observational study. Lancet Respir Med. 2020;8(5): 475-81.

18. Guan W-j, et al. Clinical characteristics of coronavirus disease 2019 in China. N Engl J Med. 2020;382(18):1708-20.

19. Sama IE, et al. Circulating plasma concentrations of ACE2 in men and women with heart failure and effects of renin-angiotensin-aldosterone-inhibitors. Eur Heart J. 2020;41(19):1810-7.
20. Shastri A, et al. Delayed clearance of SARS-CoV2 in male compared to female patients: high ACE2 expression in testes suggests possible existence of gender-specific viral reservoirs. MedRxiv. 2020.

21. Cen Y, et al. Risk factors for disease progression in patients with mild to moderate coronavirus disease 2019 - a multi-centre observational study. Clin Microbiol Infect. 2020;26(9):1242-7.

22. Cavagna $\mathrm{L}$, et al. Calcineurin inhibitor-based immunosuppression and COVID-19: results from a multidisciplinary cohort of patients in northern Italy. Microorganisms. 2020;8(7):977.

23. Rodriguez-Cubillo B, et al. Should cyclosporine be useful in renal transplant recipients affected by SARS-CoV-2? Am J Transplant. 2020;20(11):3173-81.

24. Cour M, Ovize M, Argaud L. Cyclosporine A: a valid candidate to treat COVID-19 patients with acute respiratory failure? Springer; 2020.

25. Molyvdas A, Matalon S. Cyclosporine: an old weapon in the fight against coronaviruses. Eur Respir Soc. 2020;56(5):2002484.

26. Chen $\mathrm{C}$, et al. Thalidomide combined with low-dose glucocorticoid in the treatment of COVID-19 pneumonia. 2020.

27. Russell CD, Millar JE, Baillie JK. Clinical evidence does not support corticosteroid treatment for 2019-nCoV lung injury. Lancet. 2020;395(10223):473-5.

28. Lamontagne F, et al. Corticosteroid therapy for sepsis: a clinical practice guideline. BMJ. 2018;362:k3284. https://doi.org/10.1136/ bmj.k3284.

Publisher's Note Springer Nature remains neutral with regard to jurisdictional claims in published maps and institutional affiliations. 